\title{
MORFOLOGIA DE LOS ESTADIOS INMADUROS DE LUTZOMYIA WALKERI (NEWSTEAD, 1914) (DIPTERA: PSYCHODIDAE)
}

\author{
CRISTINA FERRO, ALBERTO MORALES, EMILIO CURA
}

\begin{abstract}
Se describen los estadios inmaduros de Lu. walkeri (Newstead, 1914) (Diptera: Psychodidae). El huevo y la larva son similares a los de Lu. bahiensis, Lu. lenti y Lu. migonei. La pupa muestra una más grande similitud con la de Lu. lenti, particularmente en la desigual longitud de las cerdas del par 10 . El aspecto general de la larva corresponde a una especie que, de acuerdo a Hanson (1968), sus larvas permanecen por debajo del substrato de su comida en los cultivos o colonias de laboratorio y probablemente también sucede lo mismo en su habitat natural. Infortunadamente, los estadios inmaduros no han sido encontrados en condiciones naturales.

Las descripciones se basan en material de una colonia de Lu. walkeri que se mantiene en el Laboratorio de Entomología del Instituto Nacional de Salud de Bogotá, Colombia, S.A.
\end{abstract}

\section{INTRODUCCION}

A pesar de que son muchas las especies de Lutzomyia (Diptera: Psychodidae) conocidas en el Nuevo Mundo, hay pocas menciones de los estadios inmaduros y sólo las larvas de 54 especies de Lutzomyia han sido descritas ${ }^{(1-2)}$. De las 20 especies conocidas del Grupo migonei, dentro del cual está incluída $L u$. walkeri, las únicas descripciones que se han hecho de los estadios inmaduros son las de Lu. migonei (Barreto, 1941), Lu. lenti (Sherlock \& Carneiro, 1963) y la pupa de Lu. tupynambai (Carneiro \& Sherlock, 1964).

Las principales razones para esta falta de conocimiento son la dificultad en hallar las larvas y pupas en su habit natural y lo laborioso y el mucho tiempo que se debe emplear en la cría de los flebotomíneos ${ }^{3-4-}$ 5-6).

Sin embargo, recientemente se han desarrollado técnicas para cría de flebotomíneos en el laboratorio ${ }^{(7-8)}$ que facilitarán la producción de huevos, emergencia de larvas y su desarrollo hasta el 40 . estadio, el cual es el más importante dentro de los estadios inmaduros, para fines taxonómicos. Estas técnicas también permitirán aumentar los conocimientos actuales acerca de la pupa, la cual es quizás la menos conocida de los estadios inmaduros de los Lutzomyia.

Teniendo en cuenta la similitud entre larvas de especies cercanas, el conocimiento de los estadios inmaduros es una herramienta muy importante para la clasificación de los flebotomíneos.

El propósito de esta publicación es el de comunicar la descripción de la morfología de los estadios inmaduros de Lutzomyia walkeri (Newstead, 1914).

\section{MATERIAL Y METODOS}

Veinte huevos, quince larvas de cuarto estadio y nueve pupas fueron obtenidos de la colonia de Lutzomyia walkeri (generación 13), la cual es mantenida en el Laboratorio de Entomología del Instituto Nacional de Salud en Bogotá, Colombia ${ }^{(9)}$.

Grupo de Entomología, Instituto Nacional de Salud, Bogotá, Colombia, S.A., A.A. 80080 - 80334. 
Para medir los huevos, éstos fueron colocados estando frescos en porta-objetos de vidrio sobre papel de filtro y examinados bajo microscopio de luz.

Las larvas de cuarto estadio y las pupas fueron muertas introduciéndolas en agua tibia $\left(60^{\circ} \mathrm{C}\right)$; se hizo así porque se notó que cuando se usaba agua hirviendo las larvas perdían cerdas.

Para aclarar las larvas y pupas se colocaron en una solución de $\mathrm{KOH}$ al $20 \%$ en agua, se calentaron (en la solución) a $40-50^{\circ} \mathrm{C}$ por 5 minutos, se dejaron enfriar a temperatura ambiente y luego se transfirieron sucesivamente a alcohol $70 \%$ por $2-3$ semanas, fenol 80 $90 \%$ en alcohol absoluto por 6-24 horas y se montaron individualmente sobre una lámina, en una gota de una mezcla de Bálsamo del Canadá y fenol (1 parte de bálsamo por 3 partes de fenol); en los bordes de la gota, lejos de los especímenes se colocaron 4 pequeños pedazos de vidrio muy delgado para evitar que la laminilla comprima la preparación, se dejó secar por 48 horas y entonces se colocó la laminilla sobre la preparación exactamente sobre los pedacitos de vidrio y se agregó por uno de los extremos de la laminilla un poco de la mezcla Bálsamo del Canadá/fenol hasta que la mezcla quedó distribuída uniformemente debajo de la laminilla.

Las medidas de los huevos, larvas y pupas fueron tomadas utilizando un microscopio de luz y expresadas en micras pero el largo total del cuerpo de las larvas y pupas y el largo de la cerda caudal de la larva fueron expresados en milímetros. Los dibujos de todos los estadios fueron realizados con la ayuda de una cámara lúcida. En los dibujos que muestran los aspectos dorsal y ventral de las larvas de 4o. estadio, se ilustran sobre el lado izquierdo las cerdas y sobre el derecho el correspondiente orden numérico. Sin embargo, fue necesario mostrar las cerdas laterales 4 y 10 y la abdominal 2 sobre el lado derecho para presentar la quetotaxia de una manera más didáctica.

\section{RESULTADOS}

\section{HUEVO (Fig. 3B)}

El promedio de longitud de los 20 huevos medidos fue de $232 \mu \mathrm{m}\left(182 \mu \mathrm{m}^{-} 261 \mu \mathrm{m}\right)$ y el promedio del ancho fue de $68 \mu \mathrm{m}\left(56 \mu \mathrm{m}^{-} 82 \mu \mathrm{m}\right)$. A pesar de que los huevos oscurecen un poco después de la postura, el color permanente del huevo es marrón claro.

El interés por la morfología de los huevos de los flebotomíneos ha venido en aumento desde la primera descripción hecha por Howlett ${ }^{(10)}$. Recientemente Ward \& Ready ${ }^{(11)}$ demostraron que el diseño o decorado del huevo puede ser útil como característica taxonómica adicional; sin embargo, el decorado del corion no es necesariamente privativo de una sola especie. La estructura básica del diseño o decorado del corion de los huevos de Lutzomya walkeri vista al microscopio de luz, es poligonal. En otras especies puede presentarse en forma de cadenas paralelas, montañas o cráteres.

Ward \& Ready ${ }^{(11)}$, basados en el trabajo de Hinton $^{(12)}$, establecieron una relación entre el modelo del decorado del corion, el plastron respiratorio y el microhabitat en el cual los huevos de los flebotomíneos son depositados.

\section{LARVA (Cuarto estadio) (Figs. 1 y 2) Tabla 1.}

Quince larvas de 4o. estadio fueron medidas usando las mismas directrices empleadas por Ward ${ }^{(1)}$.

El promedio de la longitud del cuerpo fue de 2.40 $\mathrm{mm}$. $(1.80-2.80 \mathrm{~mm}$.). El cuerpo es de color casi blanco. La cabeza y la esclerita dorsal del octavo segmento abdominal son de color castaño oscuro. El $1 / 4$ distal del noveno segmento abdominal y la base de las cerdas caudales son mucho más oscuras que la esclerita dorsals Todo el cuerpo está cubierto por pequeñas espinas piramidales. Las cerdas ramificadas son de color amarillo-dorado y las cerdas en forma de espina son de color castaño. Como regla general, las cerdas en los segmentos abdominales anteriores se implantan hacia la región distal de cada segmento pero a medida que se avanza hacia los segmentos abdominales posteriores las cerdas se van implantando cada vez más hacia la región basal de cada segmento. La longitud de las cerdas se muestra en la tabla No. 1.

Cabeza (Fig. 1 - Tabla 1.)

La cápsula de la cabeza es de forma ovoide y de color castaño oscuro. Tiene en promedio una longitud de $238 \mu \mathrm{m}(198-270 \mu \mathrm{m})$ y de ancho un promedio de $171 \mu \mathrm{m}(165-181 \mu \mathrm{m})$. 


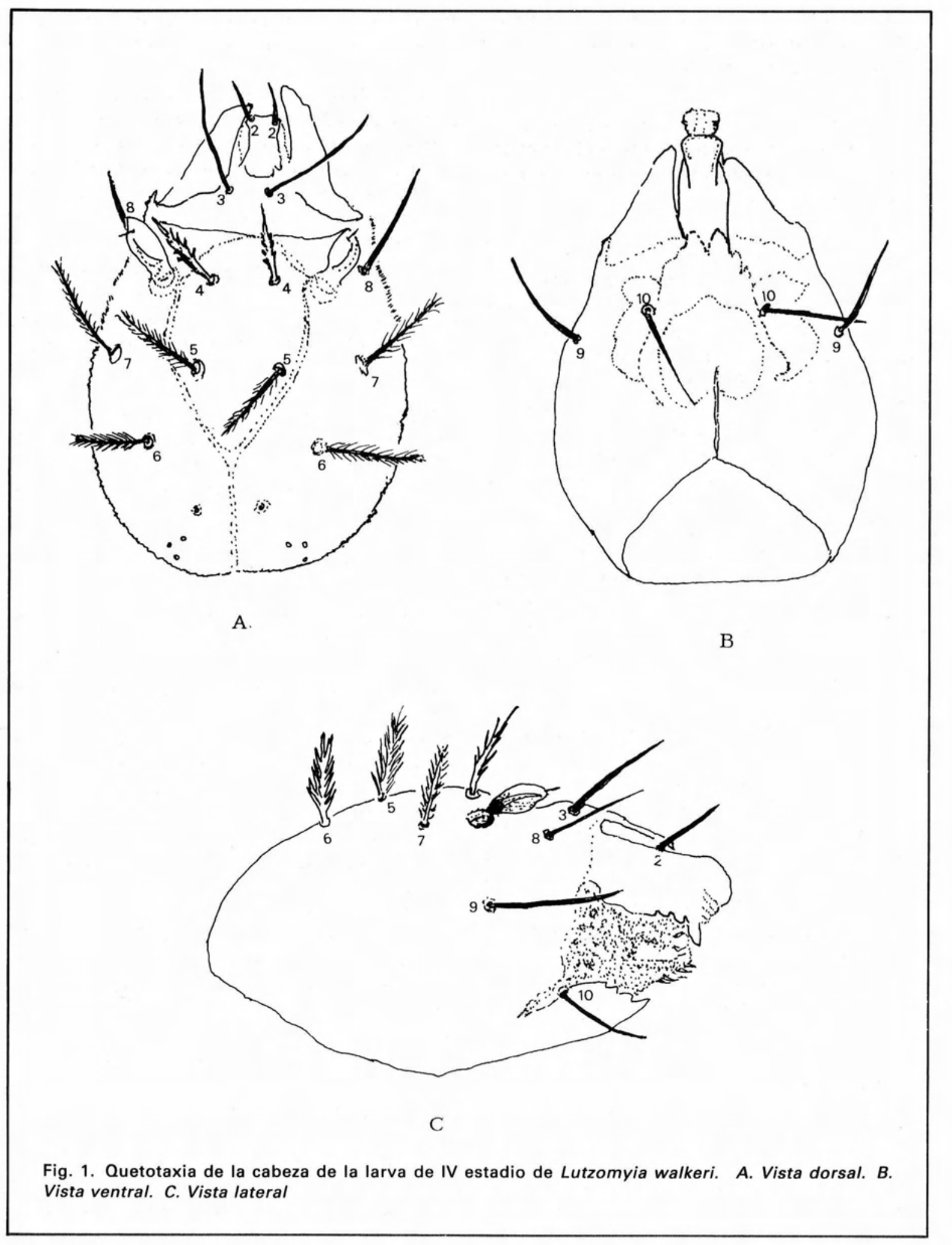


MORFOLOGIA DE LOS ESTADIOS INMADUROS DE LUTZOMYIA WALOUERI (NEWSTEAD, 1914) (DIPTERA: PSYCHODIDAE)

TABLA 1. Promedios y rangos, en un $\mu \mathrm{M}$, de la longitud de las cerdas de 15 larvas de 4o. estadio de Lutzomyia walkeri

\begin{tabular}{|c|c|c|c|c|c|c|c|c|}
\hline \multirow{2}{*}{ Cerdas } & \multirow{2}{*}{ Cabeza } & \multirow{2}{*}{ Protorax } & \multirow{2}{*}{ Mesotorax } & \multirow{2}{*}{ Metatorax } & \multicolumn{4}{|c|}{ Segmentos abdominales } \\
\hline & & & & & $T$ & 7 & 8 & 9 \\
\hline 1 & $\begin{array}{c}5 \\
(3-10)\end{array}$ & $\begin{array}{c}66 \\
(49-92)\end{array}$ & - & - & - & - & - & . \\
\hline 2 & $\left(10^{20}-26\right)$ & $\begin{array}{c}57 \\
(49-79) \\
\end{array}$ & - & - & $\begin{array}{c}27 \\
(23-33)\end{array}$ & $\begin{array}{c}27 \\
\left(16^{-33}\right)\end{array}$ & - & - \\
\hline 3 & $\begin{array}{c}74 \\
(66-82) \\
\end{array}$ & $\begin{array}{c}71 \\
(49-86) \\
\end{array}$ & - & - & - & - & - & - \\
\hline g & - & $\begin{array}{c}18 \\
(13-26)\end{array}$ & $\begin{array}{c}11 \\
(5-26)\end{array}$ & $\begin{array}{c}7 \\
(3-10)\end{array}$ & - & - & - & - \\
\hline 4 & $\begin{array}{c}58 \\
(33-82)\end{array}$ & $\begin{array}{c}60 \\
(40-79)\end{array}$ & $\begin{array}{c}50 \\
(33-82)\end{array}$ & $\begin{array}{c}56 \\
(40-66)\end{array}$ & $\begin{array}{c}48 \\
(23-56)\end{array}$ & $\begin{array}{c}60 \\
(33-73)\end{array}$ & $\begin{array}{c}54 \\
(36-66)\end{array}$ & $\begin{array}{c}38 \\
(23-53)\end{array}$ \\
\hline 5 & $\begin{array}{c}57 \\
(49-79)\end{array}$ & $\begin{array}{c}69 \\
(56-89) \\
\end{array}$ & - & - & - & - & - & - \\
\hline 6 & $\begin{array}{c}53 \\
(33-71)\end{array}$ & $\begin{array}{c}65 \\
(49-84)\end{array}$ & - & - & - & - & - & - \\
\hline 7 & $\begin{array}{c}56 \\
\left(40^{-73}\right)\end{array}$ & $\begin{array}{c}66 \\
(53-92) \\
\end{array}$ & $\begin{array}{c}80 \\
(102-49)\end{array}$ & $\begin{array}{c}106 \\
(89-122) \\
\end{array}$ & $\begin{array}{c}117 \\
(82-142)\end{array}$ & $\begin{array}{c}107 \\
(66-132)\end{array}$ & $\begin{array}{c}58 \\
(46-96) \\
\end{array}$ & $\begin{array}{c}50 \\
(16-66) \\
\end{array}$ \\
\hline 8 & $\begin{array}{c}67 \\
(53-76)\end{array}$ & $\begin{array}{c}65 \\
(40-92) \\
\end{array}$ & $\begin{array}{c}85 \\
(66-99)\end{array}$ & $\begin{array}{c}103 \\
(60-119) \\
\end{array}$ & $\begin{array}{c}115 \\
(86-132) \\
\end{array}$ & $\begin{array}{c}113 \\
(82-142) \\
\end{array}$ & $\begin{array}{c}100 \\
(73-119)\end{array}$ & $\begin{array}{c}106 \\
(49-139) \\
\end{array}$ \\
\hline 9 & $\begin{array}{c}62 \\
(49-79)\end{array}$ & $\begin{array}{c}62 \\
(40-79)\end{array}$ & $\begin{array}{c}65 \\
(36-82)\end{array}$ & $\begin{array}{c}86 \\
(53-115)\end{array}$ & $\begin{array}{c}92 \\
(59-122)\end{array}$ & $\begin{array}{c}105 \\
(66-132)\end{array}$ & $\begin{array}{c}88 \\
(53-106)\end{array}$ & $\begin{array}{c}52 \\
(23-73) \\
\end{array}$ \\
\hline 10 & $\begin{array}{c}38 \\
(31-49)\end{array}$ & $\begin{array}{c}38 \\
(23-49)\end{array}$ & $\begin{array}{c}8 \\
(7-10)\end{array}$ & $\left(7^{10}-33\right)$ & $A$ & A & $\begin{array}{c}20 * \\
(16-33)\end{array}$ & - \\
\hline 11 & - & $\begin{array}{c}50 \\
(20-76)\end{array}$ & $\begin{array}{c}38 \\
(16-53)\end{array}$ & $\begin{array}{c}39 \\
(20-49)\end{array}$ & $\begin{array}{c}10 \\
(7-13)\end{array}$ & $\begin{array}{c}9 \\
(7-13)\end{array}$ & $(16-46)$ & $\begin{array}{c}52 \\
(36-66)\end{array}$ \\
\hline 12 & - & $\begin{array}{c}8 \\
(3-16)\end{array}$ & $\begin{array}{c}7 \\
(3-16)\end{array}$ & $\begin{array}{c}7 \\
(3-16) \\
\end{array}$ & $\begin{array}{c}10 \\
(7-16)\end{array}$ & $(5-16)$ & $\begin{array}{c}8 \\
(3-13)\end{array}$ & $\begin{array}{c}21 \\
(10-33)\end{array}$ \\
\hline 13 & - & $(13-49)$ & $(16-49)$ & $\begin{array}{c}36 \\
(20-49)\end{array}$ & - & - & - & - \\
\hline 14 & - & $\begin{array}{c}6 \\
(3-10)\end{array}$ & $\begin{array}{c}11 \\
(7-16)\end{array}$ & $\begin{array}{c}11 \\
(7-16)\end{array}$ & - & - & - & - \\
\hline 15 & - & $\begin{array}{c}33 \\
(20-56)\end{array}$ & $\begin{array}{c}29 \\
(20-43)\end{array}$ & $\begin{array}{c}31 \\
\left(20^{3}-16\right)\end{array}$ & $\begin{array}{c}33 \\
(26-43)\end{array}$ & $\begin{array}{c}43 \\
\left(33^{-66)}\right.\end{array}$ & $(13-49)$ & $\left(10^{14}-23\right)$ \\
\hline
\end{tabular}

Simbolos: - No existe esta cerda en este segmento.

$A=$ Ausente (Ward, 1976b)

$\mathrm{a}=$ Cerda accesoria

* Cerca al espiraculo posterior

Las antenas se desprenden aproximadamente al nivel de la cerda 4 y se dirigen hacia adelante, son incoloras, tienen en promedio una longitud de $32 \mu \mathrm{m}$ $(30-40 \mu \mathrm{m})$ y no tienen tubérculo antenal. El primer segmento antenal es corto e inconspicuo, mide $3 \mu \mathrm{m}$ de longitud; el segmento apical de la antena es más ancho, de forma ovoide, su longitud es en promedio de $31 \mu \mathrm{m}(30-33 \mu \mathrm{m})$ y posee una cerda de $7 \mu \mathrm{m}$ de longitud.

Las cerdas 1 y 2 de la cabeza tienen forma de espina y fuecon medidas a pesar de su limitado valor taxonómico. La cerda 3 es similar a las cerdas 1 y 2 pero más larga, en forma de aguja y color castaño-oscuro. Las cerdas 4, 5, 6 y 7 son ramificadas; las cerdas 8 , 9 y 10 son como agujas y de color castaño-oscuras.
Dos grupos triangulares de manchas se presentan en la unión del vertex con el occipucio.

\section{Protórax (Fig. 2 - Tabla 1.)}

Las cerdas 1 a 11,13 y 15 son amarillas o doradas y ramificadas. Las cerdas 12 y 14 son ramificadas y relativamente pequeñas. La cerda accesoria "a" tiene forma de aguja y está localizada muy cerca a la cerda 4 .

\section{Mesotórax y Metatórax (Fig. 2 - Tabla 1.)}

Las cerdas 7 a 15 son similares a las del protórax. Las cerdas laterales 4 y 10 están más cercanas una a otra que en el protórax. La cerda 10 es mucho más pequeña. La cerda accesoria "a" es similar a la del protórax y está localizada entre las cerdas 4 y 10. 


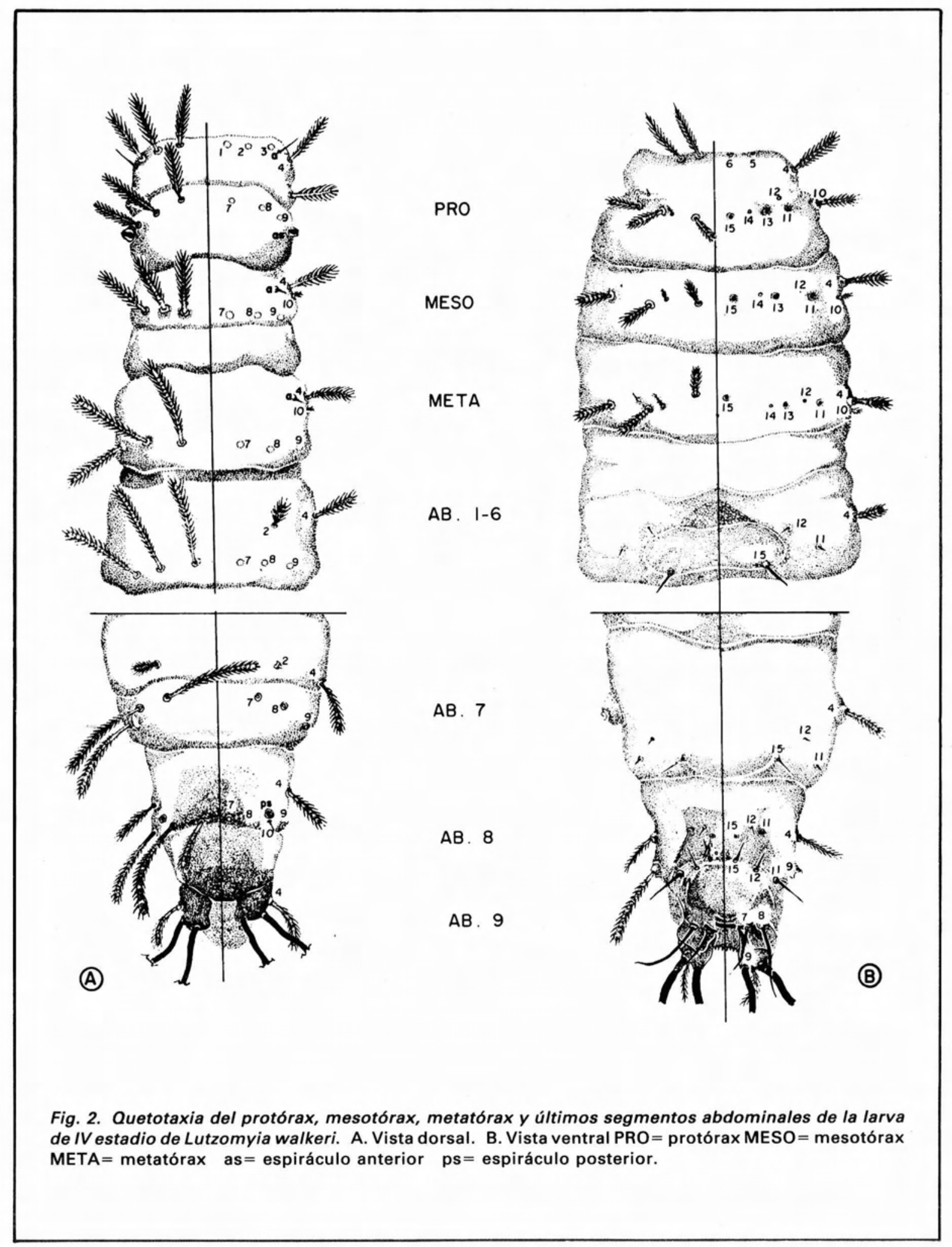


Segmentos abdominales 1 a 7 (Fig. 2 - Tabla 1.)

La cerda abdominal 2 es homóloga con la cerda protorácica 2 , de color amarillo y ramificada, pero el tamaño es menor. La cerda 4 es similar a las de los segmentos torácicos. Las cerdas 7, 8 y 9 son también similares a las del tórax pero más largas y más delgadas; la cerda 10 no existe. Las cerdas 11 y 12 son homólogas con las 11 y 12 de los segmentos torácicos pero la 11 es mucho más corta, de color castaño oscuro y se presenta en forma de espina. Las cerdas 13 y 14 están ausentes. La cerda 15 se desprende del margen del pseudopodium, tiene forma de espina y su color es castaño oscuro.

\section{Segmento abdominal 8 (Fig. 2 - Tabla 1.)}

La cerda 4 es similar a la de los segmentos torácicos y abdominales 1 a 7 . Las cerdas 7, 8 y 9 en este segmento se encuentran casi sobre el borde que limita con el segmento abdominal 9. La cerda 7 es más corta que en los otros segmentos abdominales. La pequeña cerda en forma de espina de color castaño oscuro que está cerca al espiráculo posterior fue designada como cerda 10. Las cerdas 11,12 y 15 son pequeñas y semejantes a las de los segmentos 1 a 7 .

\section{Segmento abdominal 9 (Fig. 2 - Tabla 1.)}

Las cerdas 4 y 9 son de color amarillo y ramificadas. Las cerdas 7 y 8 en forma de espina muy largas y de color castaño oscuro están colocadas debajo de las cerdas caudales. Las cerdas 11,12 y 15 tienen forma de espina y color castaño oscuro; la cerda 11 es más larga y fuerte que las 12 y 15 . El promedio de la longitud de la cerda caudal es de $0.92 \mathrm{~mm}$. (0.74 $1.02 \mathrm{~mm}$.).

\section{PUPA (Fig. 3A)}

Los caracteres distintivos de más valor para fines taxonómicos en la pupa son la forma y tamaño de las cerdas prealares y de los tubérculos mesonotales; la longitud y volumen de la pupa es importante porque corresponde al tamaño del respectivo adulto ${ }^{(13)}$. Las cerdas de la cabeza, tórax (excepto prealares) y las abdominales son muy similares en especies muy diferentes por lo tanto su valor taxonómico es muy limitado.

En promedio el largo total de la pupa es de 1.55 $\mathrm{mm}$. (1.46 - $1.61 \mathrm{~mm}$.). Los tubérculos mesonotales son de color amarillo y están cubiertos con pequeñas pápulas con longitud de $31 \mu \mathrm{m}(16-40 \mu \mathrm{m})$. La porción superior del protórax y del mesotórax están también cubiertos con pequeñas pápulas.

La cabeza tiene 10 pares de cerdas designadas de acuerdo con la quetotaxia preconizada por Forattini(6). Ellas son generalmente cortas y en forma de espina que van desde un par fino y delgado (cerdas $0.1-0.2$ de Forattini) a uno grueso y corto (6a). El par $2 b$ es espatulado. El largo de todas las cerdas es menor a $20 \mu \mathrm{m}$. Los pares más evidentes son los 3 y 5 que son los más largos y miden cerca de $20 \mu \mathrm{m}$ seguidos por los pares $0.2,2 \mathrm{a}$ y $2 \mathrm{~b}$ los cuales miden alrededor de $16 \mu \mathrm{m}$; los pares 1 y 4 tienen una longitud aproximada de $10 \mu \mathrm{m}$. Los más cortos son los pares $6 \mathrm{a}$ y $6 \mathrm{~b}$ (no medidos).

El protórax tiene 9 pares de cerdas de los cuales los pares 7, 8 y 9 tienen forma de espina, son fuertes y arrancan de un tubérculo, miden de longitud entre 10 y $20 \mu \mathrm{m}$. Los otros 6 pares son mas bien cortos y también con forma de espina y están situados en la región anterior del protórax, muy cerca a la cabeza ${ }^{(14)}$ (estos pares no fueron medidos).

El mesotórax tiene 5 pares de cerdas incluyendo la cerda prealar (par 10 de Forattini); un par desigual de cerdas se desprende de un tubérculo sencillo. La más larga cerda prealar es-espatulada y tiene una longitud en promedio de $118 \mu \mathrm{m}(99-123 \mu \mathrm{m})$ y la más corta es fina con una longitud de aproximadamente $11 \mu \mathrm{m}$ (medida en sólo 2 pupas debido a la dificultad en observarla). Los otros 3 pares de cerdas, 11A, 11B y $11 \mathrm{C}$ son en forma de espinas.

El metatórax tiene 5 pares de cerdas. Los pares 12A, 12B y 12C tienen forma de espina y arrancan de un pequeño tubérculo; los pares 13A y 13B son finas espinas y más bien cortos. Las cerdas metatorácicas no fueron medidas.

Aunque los segmentos abdominales, con la excepción del segmento 9 , tienen al menos 4 pares de cerdas dorsales sobre todos los segmentos y 4 pares de cerdas ventrales sobre los segmentos 3 a 7 , las únicas cerdas estudiadas fueron el par dorsal (par 1 de Forattini), el más importante para fines taxonómicos; estas cerdas tienen forma de espinas y se desprenden de un tubérculo notable, miden $16.50 \mu \mathrm{m}$. 


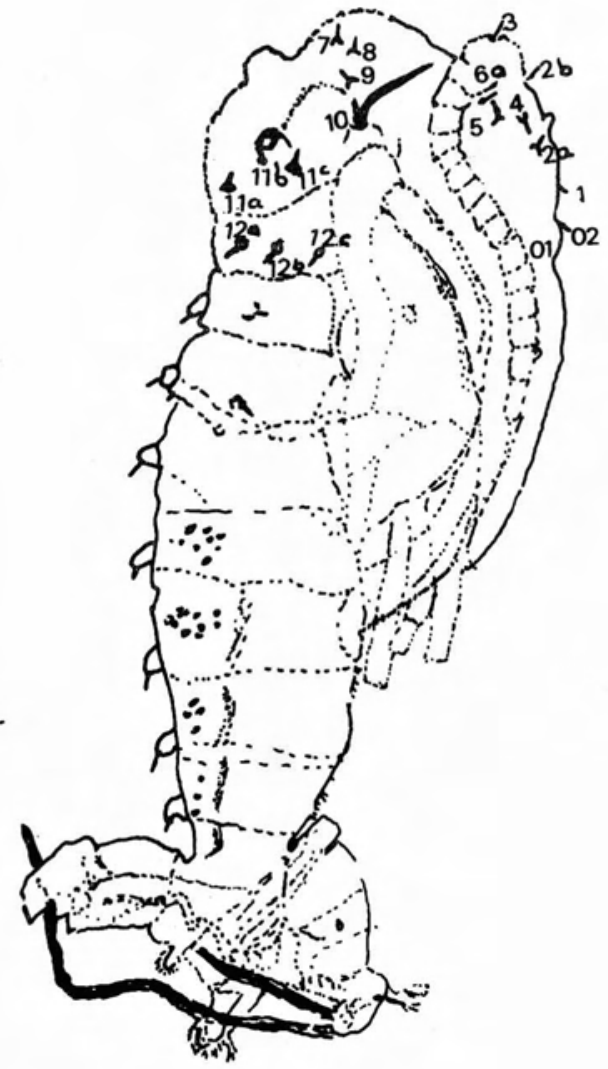

A

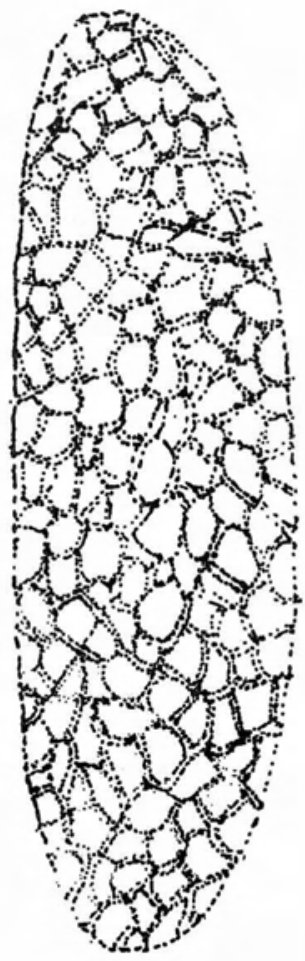

B

Fig. 3. Vista lateral de la pupa y huevo de Lutzomyia walkeri A. Pupa. B. Huevo.

\section{DISCUSION}

La quetotaxia numérica usada aquí para la larva de cuarto estadio de $\mathbf{L u}$. walkeri está basada en la usada por Ward ${ }^{(1)}$ la cual consideramos más práctica que la utilizada por Barreto ${ }^{(3)}$ o la de Hanson ${ }^{(4)}$; la usada por Ward facilita la homología de las cerdas de los diferentes segmentos. Abonnenc ${ }^{(14)}$ y Forattini( ${ }^{(6)}$ usaron sistemas similares a los de Ward ${ }^{(1)}$.
Nuestro estudio confirma que esta quetotaxia numérica es útil para otras especies además de las de subgénero Psychodopygus y grupo interme$\mathrm{dia}^{(1)}$. La cerda de forma de espina del segmento abdominal VIII, cercana al espiráculo posterior y que hemos denominado cerda 10 es homóloga con la cerda presente sobre todos los segmentos torácicos pero ausente de todos los segmentos abdominales excepto el octavo. 
La larva de $\mathrm{Lu}$. walkeri posee antenas cortas sin tubérculos antenales; el cuerpo es cilíndrico y de color pálido excepto por la cápsula cefálica, la esclerita dorsal del segmento abdominal VIII y la peinilla del segmento abdominal IX las cuales son castaño oscuras. La longitud de las cerdas caudales corresponde a menos de la mitad de la longitud total del cuerpo (del margen anterior de la cápsula de la cabeza a la "peinilla" margen posterior del segmento abdominal IX). Esta descripción corresponde a una larva que de acuerdo con Hanson ${ }^{(4)}$, excaba y permanece por debajo de la comida en los cultivos de laboratorio y probablemente haga lo mismo en su habitat natural en el suelo; esto es constante en nuestras observaciones de las larvas de colonia en el laboratorio.

Las larvas de $\mathrm{Lu}$. walkeri no han sido encontradas aún en condiciones naturales; los adultos son ocasionalmente capturados con cebo humano en área silvestre boscosa, pero más a menudo en casas rurales que están situadas a alguna distancia del bosque.

Actualmente la clasificación de los flebotomíneos está basada fundamentalmente en el estudio de la morfología de los adultos ${ }^{(15-16)}$ y en el análisis isoenzimático ${ }^{(17)}$. En el futuro un mejor conocimiento de los estadios inmaduros también contribuirá a resolver problemas taxonómicos en este grupo de insectos. El exocorion de los huevos de $L u$. bahiensis, Lu. lenti, $L u$. migonei y $L u$. walkeri tiene un decorado o diseño poligonal y las larvas de estas especies tienen el mismo aspecto general y todas tienen la misma forma de cerdas y antenas.

La pupa de $L u$. walkeri muestra una gran similitud con la de $\mathrm{Lu}$. lenti, particularmente en que ambas tienen una desigual longitud del par de cerdas 10 .

\section{SUMMARY}

The immature stages of $\mathrm{Lu}$. walkeri (Newstead, 1914) (Diptera: Psychodidae) are described. The egg and the larva are similar to those of $L u$. bahiensis, $\mathrm{Lu}$. lenti and $\mathrm{Lu}$. migonei; the pupa shows greatest resemblance to that of $L u$. lenti, particularly in the uneven length of setae pair 10 . The general aspect of the larva corresponds to a species which, according to Hanson, burrows in its food medium in laboratory cultures and probably in the soil in its natural habitat. Unfortuna- tely, the immature stages have not yet been found in nature.

\section{AGRADECIMIENTOS}

Los autores agradecen a los señores Alvaro Ricaurte, Enrique Martínez y Marco Fidel Suárez H., por su ayuda en la realización de este trabajo. Al señor Guillermo Varela por la elaboración de los dibujos y al señor Jaime Vega quien tomó las fotografías. A la señora María Herly López por su eficaz colaboración en la transcripción del manuscrito.

\section{BIBLIOGRAFIA}

1. Ward RD. The immature stages of some phelebotomine sandflies from Brazil (Diptera: Psychodidae). Syst Entomol 1976; 1:227.

2. Young DG. \& Lawyer PG. New World vectors of leishmaniasis. Current Topics in vector Research. 1987; 4:29.

3. Barretto MP. Morfología dos ovos, larvas e pupas de alguns Flebótomos de Sao Paulo. Anais Fac Med Univ Sao Paulo. 1941; 17:357.

4. Hanson WJ. The immature stages of the subfamily Phlebotominae in Panama (Diptera: Psychodidae), Ph D. thesis, University of Kansas, Lawrence, Kansas. 104 pp.

5. Guitton N. \& Sherlock IA. Descricao das fases imaturas do Phlebotomus longipalpis (Lutz \& Neiva. 1912) (Diptera, Psychodidae). Rev Brasil Biol 1969; 29 (3):383.

6. Forattini OP. Entomolgía Médica. 4o. Volume. Editora da Universidade de Sao Paulo. 1973.

7. Endris RG, Perkins PV, Young DG, Johnson RN. Techniques for laboratory rearing of sand flies (Diptera: Psychodidae). Mosq News 1982; 42(3):400.

8. Modi GB, Tesh RB. A simple technique for mass rearing Lutzomyia longipalpis and Phlebotomus papatasi (Diptera: Psychodidae) in the laboratory. J Med Entomol 1983; 20(5):568,

9. Morales A, Ferro de Carrasquilla C, e Isaza de Rodríguez C. Establecimiento de una colonia de Lutzomyia walkeri (Newstead, 1914). Biomédica. 1984; 4(1):37.

10. Howlett FM. A preliminary note on the identification of Sandflies. Bull Ent Research 1915; 6:293.

11. Ward RD, \& Ready PA. Chorionic sculpturing in some sandfly eggs (Diptera: Psychodidae). J Ent (A). 1975; 50(2): 127. 
12. Hinton HE. Respiratory systems of insect egg shells. A. Rev Ent 1969; 14:343.

13. Carneiro M. \& Sherlock IA. Estudo morfologico sobre as pupas de Phlebotominae (Diptera: Psychodidae). Rev Bras Malar Doenc Trop 1964; 16(3):311.

14. Abonnenc E. Les Phlebotomes de la Region Ethiopienne (Diptera, Psychodidae). Memoirs ORSTOM No. 55. Paris 1972.
15. Theodor O. On the classification of American Phlebotominae J Med ent 1965; 2:171.

16. Young DG. A review of the bloodsucking psychodid flies of Colombia (Diptera: Phlebotominae and Sycoracinae), University of Florida Agricultural Experimental Station Technical Bulletin 806, 1979; pp. 266.

17. The Leishmaniases. Report of a WHO Expert Committee. Technical Report series 701, 1984; pp. 70-73. 